epilepsy. Brain April 2009;132:989-998). (Respond: Prof Dr Dieter Schmidt, Epilepsy Research Group, Goethestr 5, D-14163 Berlin, Germany. E-mail: dbschmidt@t-online.de).

COMMENT. The long-term outcome of epilepsy in children may be predicted after one year of treatment. Patients with frequent seizures in the first year of treatment, especially those with symptomatic epilepsy, are at increased risk of intractable epilepsy, and should be considered for aggressive therapy.

\title{
INFLUENCE OF SCN1A GENE MUTATION ON AGE OF ONSET OF FEBRILE SEIZURES IN GEFS+
}

Twelve multigenerational families with the generalized epilepsy and febrile seizure plus (GEFS+) syndrome and a known clinically relevant epilepsy gene mutation were examined for age of onset of febrile seizures (FS or FS+), in a study at the University of Melbourne, Australia, and centers in the Netherlands and Israel. A total of 105 patients were identified with $S C N 1 A, S C N 1 B$, or $G A B R G 2$ gene mutations. Sixty-two patients presented with a FS; 43 were excluded because the FS was atypical or age of onset was imprecise. The median age of onset of FS was 12 months in subjects with SCN1A and GABRG2 mutations, and 24 months in those with $S C N 1 B$ mutation. The median age of onset in children with an $S C N 1 A$ mutation was significantly lower than for children with an $S C N 1 B$ mutation $(\mathrm{p}=0.001)$. Age of onset of FS was not significantly different in children with $S C N 1 A$ and $G A B R G 2$ mutations. In 10 families reported in the literature that had mutations available, the findings were similar to the above; median age of onset of FS in patients with $S C N 1 A$ mutations was 11 months compared to 30 months in those with $S C N 1 B$ mutations $(\mathrm{p}=0.033)$. In patients with GABRG2 mutations, age of onset was 18 months. With all families combined, the median age of onset in those with $S C N 1 A$ and $B$ mutations was 12 months and 24 months, respectively ( $\mathrm{p}<0.001)$; in those with $G A B R G 2$ mutations it was 13 months and similar to SCN1A group. (Sijben AEJ, Sithinamsuwan P, Radhakrishnan A, et al. Does a SCN1A gene mutation confer earlier age of onset of febrile seizures in GEFS+? Epilepsia April 2009;50:953-956). (Respond: Prof Ingrid E Scheffer, Director of Paediatrics, Austin Health, Neurosciences, Heidelberg, Victoria 3081, Australia.

E-mail:scheffer@unimelb.edu.au).

COMMENT. The age of onset of febrile seizures is partly dependent on the underlying gene mutation involved. In two independent cohorts of subjects with FS or FS+ and SCN1A mutations, the median age of onset was 11-12 months compared with a population median of 18 months. The proceedings of an international symposium on febrile seizures and related conditions, edited by Fukuyama Y et al, are published in Brain Dev May 2009;31(5):331-404). Nakayama J reviews the search for FS susceptibility genes in Brain Dev 2009;31(5):359-365).

The phenotypic similarities of GEFS+ and autosomal dominant FS (ADFS) are reported in a multigenerational family with febrile and afebrile seizures (Hindocha $\mathrm{N}$ et al. Epilepsia April 2009;50:937-942). The two mutations identified in families with ADFS are in genes implicated in GEFS+ (SCN1A and GABRG2). The authors conclude that it is inappropriate to separate GEFS+ and ADFS, given the clinical and genotypic overlap. 